\title{
Teaching Clinical Judgment: a Review with Consideration of Applications for Health Professions
}

\author{
Ann Gaba* \\ Hunter College, City University of New York, School of Public Health, 2180 Third Avenue, New York, NY 10035, USA
}

\begin{abstract}
This review explores the concept of clinical judgment and its relationship to critical thinking. A systematic review of the literature was performed in PubMed, using the key words "clinical judgment," and limiting the search to papers published in English. A hand search located additional pertinent documents. While papers on clinical judgment specifically in nutrition and dietetics are scarce, literature from other disciplines can inform this discussion. How these essential skills are taught and learned in the health professions, and some theoretical views of this area are examined. Following this, the underlying philosophy and educational models are discussed. Lastly, specific educational methods and strategies are described as illustrative examples of how these theoretical concepts can be applied in training health professionals.
\end{abstract}

Keywords: Clinical judgment, critical thinking, educational models, educational philosophy.

\section{INTRODUCTION}

Development and application of clinical judgment is considered essential in health professions [1]. Nutrition/ Dietetics is no exception to this. Application of the Nutrition Care Process in a variety of settings requires strong clinical judgment ability. Hackel-Smith and Leso [2] list six aspects of clinical judgment that are involved in the Nutrition Care Process. These are: collecting evidence, determining diagnosis, determining etiology, establishing goals, determining and implementing interventions. and measuring and evaluating patient outcomes. They note "including the diagnostic judgment in the nutrition care process is critical because only when a patient's nutrition problems are clearly and accurately identified will the strategies to solve them be effective". Clinical judgment includes professional ethics, enculturation to practice settings, and application of standards of practice and clinical guidelines to each patient's unique individual situation [3]. Clinical judgment utilizes both scientific knowledge and technical skills, but is more than simply the application of either or both of these. It is the acquired ability to determine how general rules and clinical guidelines apply to a given patient in a particular situation [4].

While it is clear that clinical judgment is needed, exactly what it is, and how it is acquired by practitioners in training is the subject of much discussion. Making the transition from a student's absorption of knowledge from textbooks and lectures to the professional's application of clinical judgment in practice can be a challenging endeavor. This paper will discuss the literature on teaching, learning, and applying clinical judgment in health professions.

*Address correspondence to this author at the Hunter College, City University of New York, School of Public Health, 2180 Third Avenue, New York, NY 10035, USA; E-mail: agaba@ @unter.cuny.edu
To gather information for this discussion, a systematic review of the literature was performed in PubMed, using the key words "clinical judgment," and limiting the search to papers published in English. From the articles obtained, a hand search was conducted to include relevant materials cited by the initial papers found. This was continued until no new pertinent citations could be found. While discussion of clinical judgment specifically in nutrition and dietetics is scarce, literature from other disciplines such as medicine, nursing, dentistry, and speech and language pathology were found that can inform this discussion. Following this, the underlying philosophy and educational models will be discussed. Lastly, specific educational methods and strategies are described as illustrative examples for application of these concepts in a didactic setting.

\section{WHAT IS CLINICAL JUDGMENT?}

Understanding of exactly what is meant by "clinical judgment" is tremendously complex. Clinical judgment has been described in myriad ways throughout the healthcare literature. Good clinical judgment includes not only an understanding of the diagnostic and pathophysiological aspects of an illness, but the setting in which that illness has occurred. Such judgment takes into consideration the family environment, lifestyle, and the personal, psychological, emotional, and social resources available and incorporates these into any interventions and care plans applied.

Victor-Chmil [5] explains that "critical thinking, clinical reasoning, and clinical judgment are similar and interrelated concepts. Critical thinking is the cognitive processes used for analyzing knowledge. Clinical reasoning is the cognitive and metacognitive process used for analyzing knowledge relative to a clinical situation or specific patient. Clinical judgment is the cognitive, psychomotor, and affective 
processes demonstrated through actions and behaviors". Records and Weiss [6] elaborate on this concept, indicating that clinical reasoning, diagnostic thinking, and clinical judgment are often conflated and used interchangeably. They define clinical judgment as the consideration of data from all available sources, including the clinician's past experiences, followed by the derivation of appropriate diagnoses and recommendations for intervention.

While experience is important to foster clinical judgment skills, time spent in clinical practice is, by itself, insufficient to create good clinical judgment. Tanner [1] used the term "clinical judgment" to mean "an interpretation or conclusion about a patient's needs, concerns, or health problems, and/or the decision to take action (or not), to use or modify standard approaches, or to improvise new ones as deemed appropriate by the patient's response". Redding [7] lists five cognitive skills or competencies associated with critical thinking that are part and parcel of clinical judgment: "(a) problem definition; (b) selection of supportive evidence for problem solution; (c) analysis of cause and effect relationships; (d) formation of relevant hypotheses based on values and assumptions; and (e) drawing valid conclusions based on logical consistency and validity".

Pesut [8] explains that "clinical judgments begin with an 'end' in mind," meaning that the anticipated outcomes must be part of the assessment and plan. In this view, clinical judgment is said to require four types of logic: the logic correctly identifying a diagnosis; the logic required to make care and treatment decisions that will benefit the patient; the logic by which one evaluates changes in a patient's status to evaluate the efficacy of the treatment; and the logic created by reflection on one's actions as a professional in the given situation.

In addition to logic and cognitive skill, other factors play a role. McNiesh [9] points out that "clinical judgment includes complex activities with human characteristics in a frequently ambiguous frame of reference, in a relatively uncontrolled environment." Cultural and social constructs influence the beliefs of individuals as to exactly what health or illness "means". Conrad and Baker [10] note that "variations in these beliefs and attitudes will inevitably impact a practitioner's clinical judgment".

\section{CRITICAL THINKING IN CLINICAL JUDGMENT}

Of the many writings on educational philosophy and the philosophy of education, training of health professionals in critical thinking and clinical judgment seems to rely over and over on the same philosophical underpinnings.

Montgomery [4] compares the reasoning necessary to provide optimal patient care to the concept of "phronesis," described by the Greek philosopher Aristotle. Phronesis was defined as the open-ended, flexible capacity of moral reasoners to determine the best course of action when the specific knowledge required to make such a decision is dependent on the specific circumstances presented. In health care that interpretive capacity can be considered to be clinical judgment. Aristotle held that experience was essential to phronesis. Internship and residency programs, from this point of view, are "hothouses for the cultivation of clinical judgment" [4]. Or, in the words of Socrates "I cannot teach anybody anything. I can only make them think."

The word "critical" is an adjective derived from the Greek noun kritikos, originally meaning "able to make judgments" [11]. In 1912 E.L. Thorndike [12] expanded upon this definition, saying "critical thinking is an increase of a person's general powers to respond well in thought and action and feeling".

How then does one acquire and then develop critical thinking ability? The thirteenth century theologian and philosopher Thomas Aquinas (ca.1225 - 1274) stated that human knowing can be based on two distinct forms. One, he called "speculative", which is the search to identify that which is true. The other, which he called "practical", is the ability to identify that which is good, or that which is the right thing to do. Aquinas believed that, logically, truth and goodness were closely related. He also described an innate capacity of human beings, which he called "synderesis", to know self-evident truths such as "good is to be done and evil is to be avoided".

Learning to put this into practice in particular clinical situations is conducted as an iterative practice with the guidance of experienced and competent instructors. [13] Gupta and Upshur [11] call this "virtue epistemology," which they describe as an understanding of the creation of knowledge that focuses more on the agency of the learner than on the nature of the knowledge acquired or the process of obtaining that knowledge. Or, as Aristotle put it (in Nicomachean Ethics Book: I, Chapter: VII): "Excellence is not a gift, but a skill that takes practice. We do not act rightly because we are excellent, in fact we achieve excellence by acting rightly."

\section{REFLECTION AND CRITICAL THINKING}

How then do practitioners develop critical thinking to guide their clinical judgment, and know this "excellence" when they have attained it? Critical thinking is the mental and intellectual work that uses reasoning and self-discipline to apply a given set of cognitive skills in a particular discipline or content area. This is in contrast to reflective thinking, which is said to entail application of "metacognition" or a level of consciousness in which an individual observes his or her own thoughts, actions and experiences, and engages in self-communication about the processes and results created thereby. Both of these modes of thinking have been used in educational, research, and practice settings to understand the means by which individuals apply clinical reasoning to the various problems they encounter [14].

Dunn and Musolino [15] explain that "Reflective thinking" is where learners are assessing what they know, what they need to know, and how they bridge the gap, while in the process of learning, or in response to learning after the fact. The educational pioneer John Dewey [16] described the concept of reflection as "the active, persistent and careful consideration of any belief or supposed form of knowledge in the light of the grounds that support it and the further consideration to which it tends". According to Dewey, three personal traits are essential to truly and 
properly engage in reflective practice. The first he called "openmindedness". This is a willingness to examine the facts presented in a situation regardless of the source(s) from which they come, to consider all the alternatives, even those that contradict one's own assumptions and beliefs, and to recognize the possibility that one's thinking may be in error. The second trait "responsibility" is the synthesis of the ideas under consideration into a coherent result that is applicable to the situation at hand. The third and final trait was "wholeheartedness". One holds and displays a sufficient level of personal integrity strength and fortitude to genuinely apply the reflective process to the totality of the experiences in the situation.

Mezirow [17] further developed the concept of reflection to include three progressive levels as one's skill at reflection develops. Level one is defined as the complete absence of reflective thought. Level two includes such basic reflections as to have an awareness of observations, decisions, and evaluations made. Level three, which Mezirow calls "critical reflection" adds to level two in that it includes consideration of one's needs for further learning and possible changes in perspective following the practice of reflection.

Schon [18] explored the role of what he called 'reflection in action' in professional practice. Schon suggested that many professions are similar in that the types of problems faced in professional practice are "messy," that is to say that they often involve complex and multi-faceted issues that do not present clearly right or wrong answers. Professional knowledge is well suited to address these issues, but it can only be truly understood as it occurs in a given specific context. This is difficult to articulate with any degree of precision, since it can not simply be stated as merely a list of necessary skills. Professional practice can not be reduced to the simple application of relevant theories, but is rather inherent in the actions of the practitioner. In addition, the present action is in part based on experience and previous actions, brought to mind in a particular situation. Schon called this process "reflection-in-action". This is as opposed to the post-hoc "reflection-on-action." Reflection-on-action takes place at times other than during a direct action by the professional. One takes some time to consider practice activities one has engaged in, to learn from the experience(s) and develop greater understanding of the action and efficacy these entailed.

Reflection-in-action may be seen as the development of hermeneutics for use in practice. Hermeneutics describe the recursive thought processes that characterizes the negotiation of fit between general and particular [4]. The hermeneutic branch of phenomenological research is a philosophical tradition that seeks to understand the workings of a culture by examining the experiences of individuals living in that culture. This includes, and attempts to explicate the "tacit knowledge" inherent in any given cultural setting. Such knowledge is shared among the members of a culture by implication, through experience and practice, rather than explicitly taught as a part of education or training. As members of the professional "culture," trainers and preceptors have previously absorbed such knowledge, and through their actions, examples, and corrections pass this on to their trainees. This internalized understanding of "the way things are" is derived from the practitioner's experience and understanding derived through the dialectical process of reflection (both in- and on-action), rather than from a set of externally verifiable "facts" to be inculcated in the learner. Kassirer [19] emphasizes the importance of learning this type of knowledge through experience in a practice setting, saying "cold logic as exemplified by the analytic approach, including probabilistic and causal reasoning, fails to account for the fact that humans are human, not silicon processors. Humans often jump to conclusions, using intuitive heuristics and reflexive rules of thumb. Gaining expertise is not easy, and it cannot be achieved passively." Experience and reflection upon experience is essential for the development of clinical judgment. Such thinking is needed to individualize care for each patient's unique needs.

\section{LEARNING FROM EXPERIENCE}

Redding [7] described the differences in practice between how experienced and inexperienced clinicians process a situation and determine appropriate actions to take. Expert clinicians are said to use an overall gestalt of pattern recognition based on fitting recognition of limited immediate information into an identifiable structure or definition based on previous experience. This manifests as a nearly simultaneous processing of the information and initiation of action. In contrast to this, inexperienced clinicians use less efficient decision making strategies, such as reviewing all possible diagnoses and interventions in an exhaustive process of elimination before any action is taken.

This rapid processing of the information available in a situation, based on skill and experience derived from practice, may seem to an observer to be based on an instantaneous intuitive "knowing" of what to do. Furthermore, such experts may have internalized this knowledge to such an extent that they find it difficult to verbalize an explanation as to how they "just know what to do" [20].

This can be frustrating to students who are unable to recognize the manifestations of signs and symptoms that differ from those given in their textbooks, identify individual variations, and notice qualitative changes in a patient's state that are all readily apparent to an experienced practitioner [1]. These distinct states of mind can make the teaching of clinical judgment challenging, especially in a contemporary health care setting. Pollard et al. [21] describe this dilemma saying that while clinical education is widely accepted as being the responsibility of practitioners involved in the delivery of health care services, this form of mentorship is fraught with difficulties. These may include staff shortages, lack of time, increasing workloads and productivity demands, role conflicts, and a lack of training for the role of educator.

Groopman [22] points out that sustained feedback from mentors helps trainees to understand both technical errors and errors in thinking and decision making. Watlog [23] and Hendricson et al. [24] both also emphasize the need for learning in practice to gain clinical judgment, and the crucial role of specific and immediate feedback to promote learning in that context. Students enter a practice setting with an array of "book smarts" that may not be linked to problem solving 
in a particular situation. Training with experienced practitioners can help them to recognize the practical utility of what they have studied. These authors note that expert judgment includes a willingness to trust one's own reasoning, and an understanding that many problems encountered in practice are not well defined with solutions that one can apply with certainty of a positive result.

\section{MODELING PROFESSIONAL DEVELOPMENT}

Like an experienced cook, who has learned by following recipes and then moved on to cooking by heart, clinical judgment includes knowing which technique to apply, and what could be modified or substituted in a given specific situation to produce good results. Experienced practitioners often utilize heuristics, or "rules of thumb" that they have learned in practice. Records and Weiss [6] define a heuristic as the "intuitive rules and strategies that may be applied to a wide variety of situations to generate useful solutions, even though a successful solution may not be assured."

A number of developmental models have been applied in the nursing literature that invoke the utilization of heuristics in expert practitioners. These may be instructive in looking at professional development in other areas of healthcare practice. Pena [25] describes the well known Dreyfus and Dreyfus model of professional development, which was created through phenomenological research on the "lived experiences" of professionals as they learned to function in their specific roles. This model includes a five-step progression including: novice, advanced beginner, competent, proficient, and expert. Each of these levels is characterized by further development of professional skills and judgment ability. At the "novice" stage, for example, a person simply follows the rules and directions provided by others. "Proficiency" is demonstrated when a person includes heuristics and intuition in developing their own plans for action. In this model, an "expert' does not depend on external sources of knowledge, but is able to perform most tasks in a manner that appears fluid and automatic, without a need for conscious deliberation in decision making

This model was adopted by Benner and other nursing educators to describe the developmental pathway in acquiring professional nursing skills and expertise. Gobet and Chassy [26] discuss these stages as applied by Benner. In the earliest stage, "novice", beginners learn through specific didactic instructions. They learn rules, formulas, and domain-specific facts. These are said to be "context free" in that they are acquired without any thought of a specific patient or situation. With concrete experience, one advances to the "advanced beginner" stage, and becomes able to apply understanding gained from that experience to a given situation. With further experience, one attains "competence" and displays increased efficacy and ability to organize actions and plans. At this stage, processes are still very conscious and deliberate. This is an opposed to the "proficiency" stage, where situations are seen holistically rather than in pieces. Proficient individuals can grasp and react to a situation intuitively, but still require some degree of analytical thinking to determine an appropriate action. Lastly, the "expert" uses a deep understanding of the situation to both understand what is happening and to make a decision as to which action to take in a way that is entirely fluid and intuitive. Practitioners may revert to practice at an earlier stage if they are placed into a novel situation, are learning a new area of practice, or if their "intuitive" action proves to be ineffective.

Although extensively cited, Benner's model is not without its critics. Hargreves and Lane [27] state that "a linear model of skill acquisition, linked to time and context is not sufficient to explain (developmental) experience." Cash [28] questions if "the concept of expertise is arbitrary; (because) it is legitimated by groups or individuals whose status is defined socially." (In other words, "it takes one to know one" is a questionable definition of expertise.) Gobet and Chassy [26] feel that in this theory "they underestimate the role played by analytic and conscious problem solving at the expert level". One may wonder if there truly is an intuitive "knowing" in the judgment of expert practitioners; or is what is called intuition more correctly identified as a rapid recollection of previously learned information that is brought forth by recognition of a pattern. Whereas Dreyfus and Dreyfus state "experts act a-rationally in a manner that defies explanation," Gardner [29] asserts that "so-called experts who claim to base their practice on tacit knowledge and intuition and who cannot or will not justify it to their peers are either fooling themselves or are acting in bad faith". Hams [30] takes a more moderate view, saying "experienced nurses are said to practice intuitively by virtue of having developed through critical thought, a deeply grounded knowledge base that can be applied in daily practice". The knowledge gained through experience and reflection that is accessed rapidly in practice situations can then be seen as a kind of intuition.

Nilsson and Pilhammer [31] looked at Dreyfus and Dreyfus model in medical training, and found a level of validity to it. Their study of the diagnostic performance of physicians with varying degrees of experience corresponded to the descriptions of clinical judgment as would be predicted by the Dreyfus and Dreyfus model. They concluded that with increased clinical experience, one is better able to grasp a particular clinical situation and respond accordingly, whether intuitively or otherwise.

In teaching clinical judgment, D'antonio et al. [32] conclude that "the most compelling experience we can give our students is to use class and clinical space as places where they can imagine themselves as practitioners". DiVitoThomas [33] adds "ideally, the goal of theoretical and practicum-based education is to infuse critical thinking as a habit of mind from the beginning of the student's education to graduation, and on to life-long learning. The student then "has the ability to know what is needed and the wisdom to apply that knowledge".

Carniero [34] notes that there may be a "discrepancy between the performance of some students in the classroom and in real life. For example, a student who can solve diagnostic problems without apparent difficulty in the classroom yet who trips up badly when faced with a patient in an outpatient clinic or, conversely, one who fails in the classroom but has a perfectly acceptable performance in real life. This phenomenon lies behind the well-known fact that some students with a poor academic performance can 
become competent practitioners, presumably because the clinical context facilitates their reasoning". Redding [7] points out the need of students in clinical courses to have exposure to practice settings. Early experience can influence the development of clinical judgment in either a positive or negative way, depending on the competence and quality of feedback from mentors. Socialization into critical thinking in this context will promote ongoing development of critical thinking skills in later life, long after formal education has finished.

How are these educational goals best accomplished? Kassirer [19] discusses adult learning theory. According to this theory, adults learn best by having a context for knowledge acquisition that fits in with previously gained knowledge and experience. Educational activities for adults ideally should be "intellectually challenging, enjoyable, respectful, and nonthreatening." Learning is optimized by exposure to real cases, with analysis and discussion in a spirit of collaborative inquiry. The instructor points out errors in reasoning or judgment and these are then discussed in the interest of promoting critical thinking and to enhance the value of the education.

\section{CLINICAL JUDGMENT AND EVIDENCE BASED PRACTICE}

Clinical judgment develops alongside the increasing bodies of work that underpin evidence based practice (EBP). Gupta \& Upshur [11] explore some reasons for and resistance to EBP. Evidence based practice has been incorporated into the accepted standards of care for most professional societies, government and regulatory agencies. With bodies of clinical knowledge becoming ever larger and more complex, there are limits to what one individual can claim to truly know from direct experience. Resistance to evidence based practice stems largely from practitioners' observed discrepancies between "best practice" guidelines given to them by authorities and the needs of individual patients, as well as the need to make decisions about care in the absence of definitive applicable evidence. Montgomery [4] notes that EBP "promises to refine knowledge and its application but not to supply complete information for every patient in each phase of any condition." In reality, EBP is not intended to take the place of clinical judgment, but rather to provide information to enhance it. Clinical judgment is essential for formulating the clinical question and, once obtained, for knowing what to do with the answer in order to care for the patient". Fesler-Birch [35] argues that blindly following clinical pathways and clinical protocols may be viewed by some as a panacea for providing quality care, but in the absence of critical thinking, this may not be so. As Elstein [36] explains, clinical judgment remains a crucial component of healthcare because not all clinical questions have an evidence-based answer. Furthermore, one must always determine if the evidence presented or guidelines set forth are applicable to the particular patient in a given situation. Or as Groopman [22] says "statistics cannot substitute for the human being before you; statistics embody averages, not individuals". Higgs et al. [37] comment that practitioners usually do not have complete or certain information at the time that decisions need to be made. While research can provide some helpful information, it is not possible to completely limit practice to areas where this evidence is complete and conclusive. Indeed more often than not this is not possible. A clinician needs to possess and apply knowledge derived from experience and critical thinking skills to provide optimal assessment and care. Clinicians need to apply a variety of skills in evaluating a problem, and examining the physiological, behavioral, and environmental determinants of that problem. This function, sometimes called "naming and framing," is integral to clinical judgment. Practitioners cope with uncertain situations by observing or recognizing that which is unusual or abnormal, that is, "naming" what they see, and then trying to understand the significance of the observed abnormality, "framing" the situation. A hallmark of expert clinical judgment is this ability to develop a level of clarity in an uncertain situation by drawing on past experience to correctly name and frame a given issue [38]. Gelhaus [39] points out that "although an infallibly rule-oriented robot seems more reliable at first view, in situations that require complex decisions like clinical practice the agency of a moral human person is more trustworthy." Avis and Freshwater [40] concur that expert clinical practice requires attention to context, to the individual idiosyncrasies presented in the case, and specific evidence gained through encountering the patient. Zwolsmon et al. [41] identify another potential barrier to adoption of evidence based practice. "Patients expect to participate in the decisionmaking process, and contemporary patient-centered medicine has made the experience of patients and their role in decision making more prominent." All of these things factor into the clinical judgment applied in a given situation to develop an appropriate plan or intervention.

Morcum [42] summarized the overall issues. Critics believe that non-analytic decision-making processes such as through tacit knowledge or intuition are too subjective. Depending on a practitioner's previous experience or ability to recognize patterns, especially when used in a nonconscious fashion, may be prone to biases and errors. However clinical judgment also includes logic, reflection, and critical thinking to support decisions made. Expert clinicians rely on all of these to determine right action in any particular instance, but most especially when applicable evidence based guidance is absent.

\section{THINKING ABOUT TEACHING}

Having examined previous thought on development of clinical judgment in health practitioners, what educational methods can be utilized to train students and promote their acquisition of clinical judgment? Levett-Jones [20] lists five "rights" essential to proper clinical reasoning: identifying the right cues, to take the right action, with the right patient, for the right reasons, at the right time. All of these are essential to conduct an assessment and devise a plan of care for any patient. To develop this set of skills, students need to be trained to identify and synthesize all available patient information and known risk factors to make a diagnosis and select the best course of action among available options.

Hendricson et al. [24] describe four educational strategies that have been associated with enhancement of critical thinking skills. These include: 1.) Asking questions 
that stimulate students' critical thinking as to how to define a clinical problem and provide and defend a rationale for their choice of care plan; 2.) Providing opportunities for students to listen to expert practitioners as they "talk through" their thinking while they solve a problem; 3.) Providing case scenarios and having students compare their strategies, decisions, and outcomes with those of expert practitioners; and 4.) Assigning writing exercises that require students to analyze problems, compare and contrast proposed interventions and plans, and to defend their decisions for these choices.

Spouse [43] reiterates the value of talk between student and instructor/ preceptor as they work together in the technique called "scaffolding". The gap between the knowledge and understanding of a student and an experienced professional is usually quite large. The professional uses vocabulary, techniques and practices that may be largely unfamiliar to the student. This can cause the student to feel confused, discouraged, or overwhelmed. Using the scaffolding strategy, the instructor helps to bridge this gap by making links to what the student already knows and what is being done in each activity in the clinical placement. Drawing the student's attention to the most relevant pieces of information through dialog at each step, the instructor facilitates the student's creation of her or his own knowledge and development. This has also been termed "proleptic instruction". Providing sufficient interactive support can encourage students to grasp skills and concepts by making sense to themselves of what they are being taught. Having the student think out loud as he or she enacts each practice can help the student to structure and make sense of what it is he or she is learning to do. This process can also help the instructor to target educational activities to the level of preparation and proficiency of each student. Scaffolded instruction is especially helpful when new concepts or skills are being introduced. The level of support provided through this highly interactive method can then be gradually removed as the student becomes more proficient and better able to continue developing in a more independent fashion.

Along with scaffolding, expert instructors can help students to recognize patterns, and look for solutions to problems in a scheme-driven way. As a means of helping students learn to think like experts, Mandin et al. [44] say 'teaching 'scheme-driven' searching to students seems to help them think like experienced clinicians earlier in their development as clinical problem solvers. Although students are not as efficient as the experts in scheme-driven searching, from the very start they are learning approaches to problems that took their mentors many years to develop". This can help to promote a more rapid development of clinical judgment, and bring the student up-to-speed" sooner in the course of training. McNiesh [9] agrees that beginners are often challenged by how to prioritize and organize all the information found in a clinical case. Mentors teaching them to recognize patterns and global clinical pictures can greatly assist them in advancing to a higher level of skill more expeditiously.

\section{PRECEPTING AND MENTORING}

Most health professions utilize an educational model that includes both classroom training and applied practical work, in an effort to impart both theoretical and practice knowledge to new practitioners. The people who take on the educator role in practice settings are often called "preceptors," and what they do "precepting." This can be a challenging task, which includes not only practical know-how, but socialization to the work setting, and the goals and ethics underlying a particular practice. Supervised practice is where the theoretical concepts learned in the classroom interface with the realities of service and care provided to patients. It is therefore crucial that students be afforded opportunities to learn alongside experienced professionals [45]. Student success in this transition has been an ongoing concern to educators, especially when students who have done well in the classroom are less successful in a practice setting [46].

Yonge et al. [47] describe a significant problem with this model, in that precepting often competes with other priorities in healthcare settings that leave less time for teaching, and especially for remediating students' learning if necessary. This can be exacerbated by staffing issues, high turn-over rates of both staff and patients, continuous changes in regulations and expectations of staff, and increasing staff workloads [48]. Being a preceptor can be very time consuming, especially in the earliest stages of training. Students who begin supervised practice without adequate clinical skills, or who lack interest or motivation in developing these, add to this burden. Yet the success of the student / preceptor relationship stems from the willingness of preceptors and staff to engage in teaching, and set a positive tone at their facilities when engaging with students.

Edmond [48] points out one of the greatest challenges to training practitioners in this traditional two-step process is that there can be a disconnect, or a general lack of understanding, between people developing academic curricula for students in a classroom, and the skills needed to fully engage in work for education and experience in practice settings. An assumption that practice skills are "just picked up" from exposure to the work place environment is misguided. Dusch [49] spoke about the need to understand what happens during the transition from being an "expert" student to beginning the cycle again as a "novice" practitioner. He points out that this occurs many times in life, but is often especially problematic for individuals who have been very successful in one area (e.g. the classroom) when they begin a new role where they are less capable (e.g. practice).

Educators should discuss this transition with students in advance to help them understand and prepare for supervised practice. For example, the methodical academic skills developed in a classroom setting may not be applicable or useful in a fast-paced practice setting. This transition can be very unsettling to students. In practice, situations and expectations change constantly, and often in unpredictable ways. A skilled practitioner can quickly adjust to the needs presented, and often seems to "just know" what to do, in ways not amenable to description in a textbook [50]. This can be confusing to a student who has not developed strong critical thinking skills and a level of flexibility in differing 
contexts. Setting clear learning objectives and goals for students can provide a framework for evaluating their work as they develop the skills to interact with patients in the situational context in a more fluid manner. As Kaldjan [51] explains, goals are not merely for the purpose of evaluating students' work, but to provide care and services that are ethical, and consistent with the needs and values of each patient. While this can seem self-evident to an experienced practitioner, it is important to explicitly point these out as a part of training. Developing the habits consistent with a patient-centered approach to care should start at the earliest stages training.

There has been some discussion as to whether learning these essential practice skills at side of a busy clinician is the best educational model for health professions. Changes to the American health care system have created faster "throughput" of patients in acute care. Patients are also of increasing levels of acuity, often with multiple comorbidities. Coupling this with ever increasing demands on staff members' time creates significant challenges for meeting the educational needs of students. In many settings, there is no longer a "shallow end of the pool" in which a novice can begin to swim [52]. The addition of the precepting role of training students to the many other expectations placed on staff can make for a sub-optimal learning environment [53]. This situation stands in contrast to the desires of students, who want "a happy, purposeful learning environment guided and regulated by a confident, considerate mentor who made students feel part of the team, and was concerned for the well-being and development of all students in his/her charge" [54].

Myall's study of nursing students [54] showed that students have clear criteria for what they look for in a preceptor. They wanted someone who maximized learning opportunities, while being helpful, understanding and supportive. They also wanted preceptors who were knowledgeable, experienced, and enthusiastic about their role, both as a practitioner and as a preceptor. Aligning the divergent characteristics and needs of students and preceptors can be challenging. There is general agreement that an optimal environment for learning and critical thinking is open, supportive, fosters inquiry and develops trust. It is devoid of threats and minimizes competitive judgments. Harsh responses to errors or questions create a climate of fear that limits students' true abilities and reduces their ability to learn from experiences. Students need to feel comfortable asking questions without fear of ridicule. Such negative responses only serve to increase a student's uncertainty and insecurity and diminish their performance and learning. Excessive criticism can create a vicious circle, in which an underperforming student is afraid or unwilling to ask the very questions that would yield the information necessary to improve. However, sensitivity and caring about students does not preclude having expectations as to their performance, or giving corrective feedback when necessary, in the interest of meeting objectives and goals [55].

Perceived negativity in a practice setting can be the result of the often sharp differences in "culture" between academic and practice environments. A more thorough preparation of students in didactic programs for the realities of practice settings would help to ease this burden in training and optimize use of the preceptors' time in the field.

Yet, as future practitioners, learning in a practice setting students obtain not only procedural knowledge, but are socialized and acculturated into the profession. In directly observing role models, patient responses and outcomes, and other learning cues they construct their own knowledge base [56]. The long tradition in practice-oriented professions of teaching through an apprenticeship model benefits the students in both practical skill development and clarifying exactly what is expected in the professional role. Even the most self-aware and reflective of students are not able to objectively evaluate their own performance in practice, and must therefore rely on a mentor to provide feedback and corrections [57]. Thus, they are able to integrate their personal knowledge base with the knowledge acquired through these work activities.

Another challenging aspect in this teaching and learning environment is the increasing role of patients in making healthcare decisions for themselves. Students may enter with extensive knowledge of science and guidelines for appropriate interventions, and be met with patients with ideas of their own, who expect to be equal partners with healthcare professionals in planning and carrying out their care. Preceptors' modeling of this role as a partner in health care with patients may contradict the traditional hierarchical authority structure that prevails in many institutions [58]. These seeming double standards may be yet another source of confusion for students.

Intergenerational differences can also significantly impact the student and preceptor relationship. Mangold [59] discusses the differences between the ways in which the "baby boomer" and "millennial" generations acquired their baseline knowledge, and the expectations that stem from those differences. Baby boomers were taught largely through lectures, in which an instructor gave them the information. In contrast, millennials have been educated with a constant access to information that must be extracted, manipulated, and transformed into applicable knowledge. These generational distinctions are further reflected in a study by Mallik and Hunt [60] who noted as an inter-generational issue that millennial age students were viewed by (gen-x and boomer) preceptors to expect much more consideration be given to their personal needs and preferences than students in previous decade. In spite of these perceived generational differences, Kramer [61] cautions that the concept of homogenous generational cohorts is a generalization, and can not be uniformly applied to every person of a given age range. Consistency is important in expectations for students, and organizational policies and procedures must be applied the same to all.

\section{APPLICATIONS}

If students were better prepared in critical thinking and clinical judgment before embarking upon supervised practice the often difficult transition from classroom to clinic would be likely to go more smoothly. Furthermore, the scare resource of preceptors' time would be conserved, possibly allowing for more students to be trained in the limited number of sites available. Given these substantial challenges, 
a discussion of some examples of methods for teaching and improving clinical judgment in didactic settings may be helpful.

"Experiential" or "active" learning strategies are among the most powerful ways to stimulate development of critical thinking and clinical judgment. Mills [62] points out "recent research has returned attention to the maxim that the person doing the teaching is far less important than how students are taught and what they are expected to do." Experiential learning focuses on what the learner does, and then how she or he reflects on the activity that has been performed. Active learning pedagogy is not usually spontaneous. Lessons must be carefully planned to create an environment where the given activities can be performed safely, and where students will have ample opportunities and prompts to reflect on what it is they have learned through the given activity. Having done this successfully, they are more likely to retain the skills and insights that they have gained [63].

Experiential pedagogy can be applied to nearly any educational goal, and has been utilized in areas as diverse as acquiring a new technical skill and social consciousnessraising. The fundamental theory behind this is the Deweyan concept that true learning comes from an application of individual experience followed by reflection on that experience. Unlike lectures or rote learning, this practice requires personal involvement by the students in the creation of their own learning [64].

Let us now look at some examples and strategies for experiential learning. Teaching and coaching on the practice of mindfulness in the context of practice assignments can be of benefit to students. In contrast to the practice of reflection, specifically "reflection-on-action" discussed earlier, mindfulness emphasizes awareness of the individual's experiences in the present moment. This can be helpful in enhancing the student's powers of observation, and development of "reflection-in-action". Traditional written assignments, such as case studies, can provide excellent learning opportunities. However, while these are a good indicator of a student's cognitive learning, they do not necessarily reflect student performance in practice [65]. Having students write critical reflection papers on content that they have read, and/or on life experiences, is one of the most basic means of teaching critical thinking. Connors [66] looked at use of a rubric to evaluate the extent of critical thinking in a writing assignment. A well-done rubric can guide students as to exactly what is expected of them in conducting an experiential exercise and reflecting upon it.

Another means of promoting self-reflection on learning are student or professional portfolios. These are collections of work that include evidence of practice and achievement. Gaba [67] provides an example of how e-portfolios have been successfully utilized in a dietetic internship program. There are many benefits of using portfolios [68]. Completion of a portfolio provides an individual with evidence of the continuity and development of their own work through a period of time. With proper coaching, students are able to self-evaluate and see their own progress. As part of a portfolio, or as a stand-alone assignment, keeping a journal to document and reflect upon pre-practice experiences can also be helpful. However caution is warranted when using portfolios as an evaluation tool, since concerns about a grade may inhibit the students' process of self-reflection [69].

In yet another example, Huang et al. [70] described how adding concept mapping to case studies enhances students' understanding of their work. Concept mapping is a means of organizing and creating a visual representation of the relationships between various ideas, activities, or projects. This can be done as part of planning and strategizing before commencing a project or program plan, or as a summary representation of what has been accomplished. The concept map itself consists of "nodes" representing each concept, activity, or piece of work, connected by uni- or bi-directional lines to represent the relationships between these. This provides an overview of each concept in the context of a larger whole, which in turn helps to stimulate critical thinking. In addition to mapping case studies, concept maps can be applied to portfolios, lesson plans, curricula, or other tasks where an overview of how all the parts fit together would be of value.

Next let us examine some specific pedagogical methods to bring experiential learning into the classroom. One method, especially useful for teaching interdisciplinary teamwork is called "jigsaw learning." In this method, students are assigned a "home" group wherein they will study a multi-faceted topic. Within each group, individuals are tasked with becoming "experts" on one aspect of the topic. Students then move into groups based on these assigned aspects. Each "expert group" studies their content area in depth. This can take place within one class session, or over a longer time frame as needed. When sufficient time has elapsed, and study work completed, each student returns to their home group with his or her "piece of the puzzle." It is then the role of the new experts to teach the members of their home group, and all together complete the jigsaw puzzle. All of the "pieces" need to be heard and understood in order to complete the assignment.

A useful technique while working through a practice learning experience is the "think aloud approach." The main idea behind the think aloud approach is for the instructor to gain access to the student's thought processes and vice versa while carrying out a task. [71, 72] When an instructor is demonstrating a task, she or he "thinks aloud" as they proceed through the actions. This reveals the otherwise hidden train of thought, and application of knowledge and the ongoing internal adjustments based on feedback within the situation that an experienced practitioner may take for granted. With a student doing the same, errors in judgment or missing information or insights can be readily identified and corrected. Some examples where this could be applied include demonstrations and "teach backs" of procedures previously observed.

A step beyond a typical classroom assignment when reallife experiences are desirable but not possible, or not available, simulations can provide a degree of practice. The more closely a simulation mimics the real situation that it is simulating, the more valuable the exercise will be. The intention of using a simulation is not to replace the important learning that takes place in supervised practice, but to enhance the student's preparation for that through a 
controlled didactic experience [73]. Some examples of simulation include use of actors to simulate interaction with a patient; simulated environments, such as a hospital room or a commercial kitchen; and computer-based simulations of electronic medical records. One benefit of simulation is the opportunity for students to repeat an exercise multiple times to gain a level of proficiency and comfort with a given activity. They also provide a safe environment for learning skills where it is not possible to harm a "real" patient [74]. An example is described by Seybert (2011) [75], who described a course for pharmacy students utilizing both computer-based and "live" patient care simulations to develop both patient care and critical thinking skills.

"Simulators" are devices and/or environments that recreate aspects of the real world encountered in practice. These imitations allow for experiential learning to take place in a more sophisticated way. A simulation is said to be "high fidelity" if it closely replicates the experience being simulated. Since there are now available mannequins that display a wide range of human responses, many high fidelity simulation scenarios are constructed around their use. Realistic environments add to the sense of authenticity in these exercises [76]. Following any simulation experience, debriefing is essential as a means of developing clinical judgment. Lederman [77] states that post-simulation debriefing should be planned and structured to encourage reflection and integration of what was experienced into each student's personal fund of knowledge. Through the debriefing process all participants make meaning of the experience(s) for themselves and together, and the instructor / facilitator then relates the insights gained to other situations that they may encounter in practice.

Although advancing technology has provided opportunities for very realistic physical-world simulation, there is a growing interest in on-line or virtual simulations. Since they do not require a physical environment, an advantage of virtual simulations is their application for asynchronous teaching/learning and incorporation into the expanding world of e-learning. Klaus [78] offers a cautionary note here, saying "the strength of e-learning is also its weakness, we confuse information with knowledge and knowledge with judgment. The clue that we need to follow depends not only on new technology but also on our oldest tool, which is human interaction." Muirhead [79] counters that "e-learning does offer an opportunity for students to participate and contribute to teaching and learning with their experiences by using discussion boards."

Clearly there is much more to develop and learn about the optimal role of these technologies and their potential for developing students' clinical judgment abilities. In one example of this, a study incorporated several of the techniques discussed above. Wooley and Jarvis [80] described a 'cognitive apprenticeship' within a simulation suite, where nursing students and instructors utilized the "think aloud" method, observed and practiced various tasks, and worked to develop their clinical judgment, critical thinking, and collaborative problem solving skills.
A novel twist on simulations are those that take the form of games. These can exist in both high tech and low tech forms. Gaming is a viable alternative to simulations based on more traditional pedagogy, which through its interactive format increases student engagement in the lesson being taught [81]. Games can be especially effective where repeated practice is needed to help reinforce knowledge [82, 83]. An example of this is provided by Stanley and Latiner [83] describing a game called "the Ward," an in-class activity designed to stimulate critical thinking in nursing students. They conclude that "games are a fundamental part of our culture, have some popularity in teaching, can reinforce critical thinking, and make learning fun for both students and tutors." In gaming, the student-player's performance is essentially self-evaluating. In a well-designed game, players know if they are winning, losing, completing a level of play, or repeating an error. This can be incorporated into classroom settings or be assigned separately. Games can be played in groups or individually and lend themselves to a variety of teaching environments and styles. This is a promising area for further development in education for professional practice.

\section{CONCLUSIONS}

Training in clinical judgment is essential for the transition of students into competent practitioners.

Because of the burdens already constraining the available preceptors, having students prepared with critical thinking skills and a degree of clinical judgment before they begin practica placements is highly desirable. Including more experiential learning, practice simulation, and other activities to begin to develop clinical judgment should begin as early as possible in didactic programs. A wide variety of educational strategies and methods exist to promote the development of clinical judgment. These can be applied with learners across the educational continuum, from students, to post-graduate supervised practice, and continuing education for practitioners. Established strategies should be more fully incorporated into dietetic education, and emerging methodology for enhancement of clinical judgment should be developed and evaluated. Having graduates who are better prepared for this aspect of supervised practice could reduce the burden on preceptors and supervisors at practice training sites.

\section{GLOSSARY:*}

\begin{tabular}{|l|l|}
\hline assessment & $\begin{array}{l}\text { gathering and organizing patient data, cues, or } \\
\text { information to determine patient status and need } \\
\text { for intervention }\end{array}$ \\
\hline clinical judgment & $\begin{array}{l}\text { weighing evidence arising in the clinical situation } \\
\text { against appropriate contextual and domain-relevant } \\
\text { knowledge [37] }\end{array}$ \\
\hline clinical reasoning & $\begin{array}{l}\text { a complex multi-dimensional cognitive process that } \\
\text { uses both formal and informal methods to } \\
\text { simultaneously gather and evaluate assessment data }\end{array}$ \\
\hline critical thinking & $\begin{array}{l}\text { self-guided, self-disciplined thinking which } \\
\text { attempts to reason at the highest level of quality in } \\
\text { a fair-minded way [84] }\end{array}$ \\
\hline
\end{tabular}




\begin{tabular}{|c|c|}
\hline debriefing & $\begin{array}{l}\text { an activity that follows a simulation experience, } \\
\text { led by a facilitator, where feedback is provided on } \\
\text { the participants' performance; the simulation } \\
\text { activities are discussed, and reflective thinking is } \\
\text { encouraged }\end{array}$ \\
\hline dialectical & $\begin{array}{l}\text { discourse between two or more people holding } \\
\text { different points of view about a subject, who wish } \\
\text { to establish the truth of the matter guided by } \\
\text { reasoned arguments }\end{array}$ \\
\hline enculturation & $\begin{array}{l}\text { the process by which an individual learns the } \\
\text { traditional content of a culture and assimilates its } \\
\text { practices and values }\end{array}$ \\
\hline epistemology & $\begin{array}{l}\text { the study of knowing; study of the nature of } \\
\text { knowledge; asking how do we know things, what } \\
\text { do we know, why we know, is what we know true, } \\
\text { and what are the limits of knowledge }\end{array}$ \\
\hline hermeneutic & $\begin{array}{l}\text { a tradition of phenomenological research that } \\
\text { suggests one can access and interpret a culture } \\
\text { through the experiences of individuals within the } \\
\text { culture [9] }\end{array}$ \\
\hline heuristic & $\begin{array}{l}\text { rule of thumb, such as recognizing a pattern, a } \\
\text { mental shortcut, or a method of processing large } \\
\text { amounts of data to reduce cognitive strain [71] }\end{array}$ \\
\hline metacognition & $\begin{array}{l}\text { a method of introspection in which one is supposed } \\
\text { to contemplate or reflect on one's own thinking } \\
\text { [19]; thinking about thinking [72] }\end{array}$ \\
\hline mindfulness & $\begin{array}{l}\text { maintaining a moment-by-moment awareness of } \\
\text { our thoughts, feelings, bodily sensations, and } \\
\text { surrounding environment }\end{array}$ \\
\hline pedagogy & $\begin{array}{l}\text { the activities of educating or instructing; activities } \\
\text { that impart knowledge or skill }\end{array}$ \\
\hline preceptor & $\begin{array}{l}\text { an expert or specialist who gives practical } \\
\text { experience and training to a student }\end{array}$ \\
\hline professionalism & $\begin{array}{l}\text { skill or behavior that goes beyond what an ordinary } \\
\text { person would have; behaving in a more formal or } \\
\text { business-like manner }\end{array}$ \\
\hline $\begin{array}{l}\text { proleptic } \\
\text { instruction }\end{array}$ & $\begin{array}{l}\text { use of a dialogue between peers to illicit and } \\
\text { transmit learning }\end{array}$ \\
\hline reflection & $\begin{array}{l}\text { being critical of one's own performance and } \\
\text { problem-solving processes and to compare them } \\
\text { with those of an expert, another learner, and } \\
\text { ultimately, an internal cognitive model of expertise } \\
\text { [80] }\end{array}$ \\
\hline scaffolding & $\begin{array}{l}\text { an educational strategy in which learning is } \\
\text { supported according to current skill level, and } \\
\text { activities are organized to assist the learner to } \\
\text { progress to the next level [80] }\end{array}$ \\
\hline tacit knowledge & $\begin{array}{l}\text { implicit information shared by a culture, what it is } \\
\text { assumed that 'everybody knows' in a culture, } \\
\text { profession, practice setting, or other group }\end{array}$ \\
\hline
\end{tabular}

* While some of these terms have been defined in various ways elsewhere, the author has included these definitions to clarify their meaning in the present text.

\section{CONFLICT OF INTEREST}

The author declares no funding for this project, and no conflict of interest.

\section{ACKNOWLEDGEMENTS}

None declared.

\section{REFERENCES}

[1] Higgs J, Burn A, Jones M. Integrating clinical reasoning and evidence-based practice. AACN Clin Issues 2001; 12(4): 482-90.

[2] Foundation for critical thinking.Available from http://www.criticalthinking.org/pages/defining-criticalthinking/766 [accessed 4/5/14].

[3] McNiesh S. Demonstrating holistic clinical judgment: preceptors perceptions of new graduate nurses. Holist Nurs Pract 2007; 21(2): 72-8.

[4] Simmons B, Lanuza D, Fonteyn M, Hicks F, Holm K. Clinical reasoning in experienced nurses. West J Nurs Res 2003; 25(6): 701-19.

[5 ] Kassirer JP. Teaching clinical reasoning: case-based and coached. Acad Med 2010; 85(7): 1118-24.

[6] Banning M. The think aloud approach as an educational tool to develop and assess clinical reasoning in undergraduate students. Nurs Educ Today 2008; 28: 8-14.

[7] Woolley NN, Jarvis Y. Situated cognition and cognitive apprenticeship: A model for teaching and learning clinical skills in a technologically rich and authentic learning environment. Nurs Educ Today 2007; 27: 73-9.

[8] Tanner CA. Thinking like a nurse: a research-based model of clinical judgment in nursing. J Nurs Educ 2006; 45(6): 203-11.

[9] Hakel-Smith N, Lewis NM. A standardized nutrition care process and language are essential components of a conceptual model to guide and document nutrition care and patient outcomes. J Am Diet Assoc 2004; 104(12): 1878-84.

[10] Nelson S. The lost path to emancipator practice: towards a history of reflective practice in nursing. Nurs Philos 2012; 13: 202-13.

[11] Ontgomery, M. How doctors think - clinical judgment and the practice of medicine. New York: Oxford University Press 2006.

[12] Victor-Chmil J. Critical thinking versus clinical reasoning versus clinical judgment: differential diagnosis. Nurs Educ 2013; 38(1): 34-6.

[13] Records NL, Weiss AL. Clinical judgment: an overview. Nat Student Speech Lang Hearing Assoc J 1993; 20: 105-11.

[14] Redding DA. The development of critical thinking among students in baccalaureate nursing education. Holist Nurs Pract 2001; 15(4): 57-64.

[15] Pesut DJ. Clinical Judgment: Foreground / Background. J Professional Nurs 2001; 17(5): 215.

[16] Conrad P, Barker KK. The social construction of illness: key insights and policy implications. J Health Soc Behav 2010; 51: S50-79.

[17] Gupta M, Upshur R. Critical thinking in clinical medicine: what is it? J Eval Clin Pract 2012; 18: 938-44

[18] Thorndike EL. Education. Macmillan: New York, 1912.

[19] Kinghorn W. Conscience as Clinical Judgment: Medical Education and the Virtue of Prudence. Am Med Assoc J Ethics 2013; 15(3): 202-5.

[20] Kuiper RA, Pesut DJ. Promoting cognitive and metacognitive reflective reasoning skills in nursing practice: self-regulated learning theory. J Adv Nurs 2004; 45(4): 381-91.

[21] Dunn L, Musolino GM. Assessing reflective thinking and approaches to learning. J Allied Health 2011; 40(3): 128-36.

[22] Dewey J. How do we think. Boston: Heath 1933.

[23] Mezirow J. A critical theory of adult learning and education. Adult Educ 1981; 32: 3-24.

[24] Schon DA. The reflective practitioner: how professionals think in action. New York: Basic Books 1983. 
[25] Levett-Jones T, Hoffman K, Dempsey J, et al. The 'five rights' of clinical reasoning: An educational model to enhance nursing students' ability to identify and manage clinically 'at risk' patients. Nurs Educ Today 2010; 30: 515-20.

[26] Pollard C, Ellis L, Stringer E, Cockayne D. Clinical education: A review of the literature. Nurs Educ Pract 2007; 7: 315-22.

[27] Jerome G. How doctors think. New York: Houghton Mifflin 2008.

[28] Watling C, Driessen E, van der Vleuten CMP, Vanstone M, Lingard L. Beyond individualism: professional culture and its influence on feedback. Med Educ 2013; 47: 585-94.

[29] Hendricson MA, Andrieu SC, Chadwick G, et al. Educational strategies associated with development of problem-solving, critical thinking, and self-directed learning. J Dental Educ 2006, 70(9): 925-36.

[30] Peňa A. The Dreyfus model of clinical problem-solving skills acquisition: a critical perspective. Med Educ 2010; 15: 3402-4846.

[31] Gobet F, Chassy P. Towards an alternative to Brenner's theory of expert intuition in nursing: A discussion paper. Int J Nurs Stud 2008; 45: 129-39.

[32] Hargreaves J, Lane D. Delya's story: from expert to novice, a critique of Benner's concept of context in the development of expert nursing practice. Int J Nurs Stud 2001; 38: 389-94.

[33] Cash K. Benner and expertise in nursing: a critique. Int J Nurs Stud 1995; 32(4): 527-32.

[34] Gardner L. From novice to expert: Benner's legacy for nurse education. Nurs Educ Today 2012; 32: 339-40.

[35] Hams SP. A gut feeling? Intuition and critical care nursing. Inten Crit Care Nurs 2000, 16: 310-8.

[36] Nilsson MS, Pilhammar E. Professional approaches in clinical judgments among senior and junior doctors: implications for medical education. BMC Med Educ 2009; 9: 25 -30.

[37] D'Antonio PO, Brennan AMW, Curley MAQ. Judgment, Inquiry, Engagement, Voice: Reenvisioning an Undergraduate Nursing Curriculum Using a Shared Decision-Making Model. J Professional Nurs 2013; 29: 407-13.

[38] DiVito-Thomas P. Identifying critical thinking behaviors in clinical judgments. J Nurs Staff Develop 2000; 16(4): 174-180.

[39] Carneiro AV. Clinical reasoning. What is its nature? Can it be Taught? Rev Port Cardiol 2003; 22(3): 433-43.

[40] Fesler-Birch DM. Critical thinking and patient outcomes: A review. Nurs Outlook 2005; 53: 59-65.

[41] Elstein AS. On the origins and development of evidence-based medicine and decision making. Inflam Res 2004; 53, Suppl 2: S144-89.

[42] Andersen DK. How can educators use simulation applications to teach and assess surgical judgment? Acad Med 2012; 87(7): 93441.

[43] Gelhaus P. Robot decisions: on the importance of virtuous judgment in clinical decision making. J Eval Clin Pract 2011; 17: 883-7.

[44] Avis M, Freshwater D. Evidence for practice, epistemology, and critical reflection. Nurs Philosophy 2006; 7: 216-24.

[45] Zwolsman S, Te Pas E, Hooft L, Wieringa-de Waard M, Van Dijk $\mathrm{N}$. Barriers to GPs' use of evidence-based medicine: a systematic review. Br J Gen Pract 2012; 62(600): e511-21.

[46] Marcum JA. An integrated model of clinical reasoning: dualprocess theory of cognition and metacognition. J J Eval Clin Pract 2012; 18: 954-61.

[47] Spouse J. Scaffolding student learning in clinical practice. Nurs Educ Today 1998; 18: 259-66.

[48] Mandin H, Jones A, Woloschuk W, Harasym P. Helping students learn to think like experts when solving clinical problems. Acad Med 1997; 72(3): 173-9.

[49] White C. A socio-cultural approach to learning in the practice setting. Nurs Educ Today 2010; 30: 794-7.

[50] Zaidi Z, Jaffery T, Shahid A, Moin S, Gilani A, Burdick W. Change in action: using positive deviance to improve student clinical performance. Adv Health Sci Educ Theory Pract 2012; 17: 95-105.

[51] Yonge O, Myrick F, Ferguson L, Lughana F. Promoting effective preceptorship experiences. J Wound Ostomy Continence Nurs 2005 ; 32(6): 407-12.
[52] Edmond CB. A new paradigm for practice education. Nurs Educ Today 2001; 21: 251-9.

[53] Disch J. From Expert to Novice. J Prof Nurs 2002; 18(6): 310.

[54] Neary M. Responsive assessment: assessing student nurses' clinical competence. Nurs Educ Today 2001; 21: 3-17.

[55] Kaidjian LC. Teaching practical wisdom in medicine through clinical judgment, goals of care, and ethical reasoning. J Med Ethics 2010; 36: 558-62.

[56] Sedgwick M, Harris S. A critique of the undergraduate nursing preceptorship model. Nurs Res Pract 2012; 12: 144-9.

[57] Neary M. Supporting students' learning and professional development through the process of continuous assessment and mentorship. Nurs Educ Today 2000; 20: 463-74.

[58] Myall M, Levett-Jones T, Lathlean J. Mentorship in contemporary practice: the experiences of nursing students and practice mentors. J Clin Nurs 2008; 17: 1834 - 42.

[59] Watling C, Driessen E, Van der Vleuten CMP, Lingard L. Learning from clinical work: the roles of learning cues and credibility judgments. Med Educ 2012; 46: 192-200.

[60] Bahn D. Social Learning Theory: its application in the context of nurse education. Nurs Educ Today 2001; 21: 110-7.

[61] Myrick F, Yonge OJ. Creating a climate for critical thinking in the preceptorship experience. Nurs Educ Today 2001; 21: 461-7.

[62] Hall WA, Developing clinical placements in times of scarcity. Nurs Educ Today 2006; 26: 627-33.

[63] Mangold K. Educating a new generation: teaching baby boomer faculty about millennial students. Nurs Educ 2007; 32(1): 21-3.

[64] Malik M, Hunt JA. Plugging a hole and lightening the burden: a process evaluation of a practice education team. J Clin Nurs 2007; 16: $1848-57$

[65] Kramer LW. Generational diversity. Am J Crit Care Nurs 2010; 29(3): 125-8

[66] Mills BJ. Active learning strategies in face-to-face courses. Idea Paper \#53. www.theideacenter.org [accessed 2/10/14].

[67] Michael J. Where's the evidence that active learning works? Advan Physiol Educ 2006; 30: 159-67.

[68] Fowler J. Experiential learning and its facilitation. Nurs Educ Today 2008; 28: 427-33.

[69] Vyas D, Ottis EJ, Caligiuri FJ. teaching clinical reasoning and problem-solving skills using human patient simulation. Am J Pharm Educ 2011; 75(9): Article 189.

[70] Connors P. Assessing written evidence of critical thinking using an analytic rubric. J Nutr Educ Behav 2008; 40: 193-4.

[71] Gaba A. Development and evaluation of an e-portfolio for use in a dietetic internship program. Proc Soc Behav Sci 2014; 2:122-3.

[72] Spence W, El-Ansari W. Portfolio assessment: practice teachers' early experience. Nurs Educ Today 2004; 24: 388-401.

[73] McMullan M. Students' perception on the use of portfolios in preregistration nursing education: a questionnaire survey. Int $\mathrm{J}$ Nurs Stud 2006; 42: 333-43.

[74] Huang YC, Chen HH, Yeh ML, YC. Case studies combined with or without concept maps improve critical thinking in hospital-based nurses: A randomized-controlled trial. Int J Nurs Stud 2012; 49: 747-54.

[75] Seropian MA. General concepts in full scale simulation: getting started. Ane Anal 2003; 97: 1695-705.

[76] Seybert AL, Kobulinsky LR, McKaveney TP. Human patient simulation in a pharmacotherapy course. Am J Pharm Educ 2008; 72(2): Article 37.

[77] Seybert AL, Kane-Gill SL. elective course in acute care using online learning and patient simulation. Am J Pharm Educ 2011; 75(3): Article 54.

[78] Hart D, McNeill MA, Griswold-Theodorson S, Bhatia K, Joing S. High fidelity case-based simulation debriefing: everything you need to know. Acad Emerg Med 2012; 19: 1084.

[79] Lederman LC. Debriefing toward a systemic assessment of theory and practice. Simul Gaming 1992; (2): 145-59.

[80] Klass DJ. Will e-learning improve clinical judgment? BMJ 2004; 328: 1147-8.

[81] Muirhead RJ. E-learning: is this teaching at students or teaching with students? Nur Forum 2007; 42(4): 178-84. 
[82] Blakely G, Skirton H, Cooper S, Allu P, Nelmes P. Use of educational games in the health professions: A mixed-method study of educational perspectives in the UK. Nurs Health Sci 2010; 12: 27-32.
[83] Blakely G, Skirton H, Cooper S, Allum P, Nelmes P. Educational gaming in the health sciences: systematic review. J Adv Nur 2008; 65(2): 259-69.

[84] Stanley D, Latimer K. 'The Ward': A simulation game for nursing students. N Educ Pract 2011; 11: 20-5.

Received: December 05, 2014

Revised: December 20, 2014

Accepted: December 29, 2014

(c) Ann Gaba; Licensee Bentham Open.

This is an open access article licensed under the terms of the Creative Commons Attribution Non-Commercial License (http://creativecommons.org/licenses/ by-nc/3.0/) which permits unrestricted, non-commercial use, distribution and reproduction in any medium, provided the work is properly cited. 